\title{
Research and Development for Spoken Language Systems
}

\author{
Victor W. Zue \\ Spoken Language Systems Group \\ Laboratory for Computer Science \\ Massachusetts Institute of Technology
}

\section{OBJECTIVE:}

The goal of this research is to develop a spoken language system that

will demonstrate the usefulness of voice input for interactive problem solving. The system will accept continuous speech, and will handle multiple speakers without explicit speaker enrollment. Combining SUMMIT, a segment-based speech recognition system, and TINA, a probabilistic natural language system, to achieve speech understanding, the system will be demonstrated in an application domain relevant to the DoD.

\section{SUMMARY OF ACCOMPLISHMENTS}

- Improved the performance and expanded the capabilities of the VOYAGER urban exploration and navigation system. Specifically:

- incorporated an $\mathrm{N}$-best interface between speech recognition and natural language processing,

- expanded the coverage of the natural language component and the back-end, and

- ported VOYAGER to a Sun workstation with a set of commercially available DSP boards, pipelined the computation, and reduced the computation time to approximately 5 times real-time.

- Developed a mechanism for automatically generating tasks in the VOYAGER framework to promote interactive problem solving by users, thus enabling us to collect spontaneous speech from users in a goal-directed mode,

- Performed acoustic and linguistic analysis on nearly 3,000 sentences, contrasting the differences between read and spontaneous speech.

- Developed the initial version of ATIS, collected pilot data, and participated in the first round of common evaluation.

\section{PLANS:}

- Improve the speech recognition performance by incorporating context-dependency in phonetic modelling.

- Fully integrate TINA and SUMMTT in order to exploit speech and natural language symbiosis.

- Continue to increase and improve the knowledge base of VOYAGER, so that correct and natural responses can be generated.

- Collect additional speech and text data during actual problem solving for system development and evaluation, and continue to evaluate the performance of VOYAGER.

- Port SUMMTT to the ATIS task, and perform overall system evaluation.

- Continue hardware development, such that the system will soon be able to run in near real-time. 